\title{
Study of PtPd Bimetallic Nanoparticles for Fuel Cell Applications ${ }^{1}$
}

\author{
Rodrigo Esparza ${ }^{a *}$, Alan Santoveña ${ }^{a}$, Alvaro Ruíz-Baltazar ${ }^{b}$, Alvaro Angeles-Pascual ${ }^{c}$, \\ Daniel Bahena ${ }^{c}$, Jose Maya-Cornejo ${ }^{a}$, Janet Ledesma-García ${ }^{d}$ Ramiro Pérez ${ }^{a}$ \\ ${ }^{a}$ Centro de Física Aplicada y Tecnología Avanzada, Universidad Nacional Autónoma de México, \\ Santiago de Querétaro, Qro., México \\ ${ }^{b}$ CONACYT, Centro de Física Aplicada y Tecnología Avanzada, Universidad Nacional Autónoma de \\ México, Santiago de Querétaro, Qro., México \\ ${ }^{c}$ Laboratorio Avanzado de Nanoscopía Electrónica - LANE, Centro de Investigación y de Estudios \\ Avanzados del Instituto Politécnico Nacional - CINVESTAV, México D.F., México \\ ${ }^{d}$ División de Investigación y Posgrado, Facultad de Ingeniería, Universidad Autónoma de Querétaro, \\ Santiago de Querétaro, Qro., México
}

Received: December 07, 2016; Revised: March 28, 2017; Accepted: April 9, 2017

\begin{abstract}
Bimetallic nanoparticles are of special interest for their potential applications for fuel cells, mainly for portable power applications. Among the bimetallic systems, Pt-Pd bimetallic nanoparticles have received great interest as they can be widely used as effective catalysts for various electrochemical reactions. In this work, Pt-Pd alloy bimetallic nanoparticles were synthesized through a chemical reduction method. The nanoparticles were characterized using aberration-corrected scanning/transmission electron microscopy (STEM). Also, parallel beam X-Ray diffraction analysis was carried out to evaluate the crystallographic structure. High-angle annular dark field (HAADF)-STEM images of the Pt-Pd bimetallic nanoparticles were obtained. The contrast of the images shows that the nanoparticles have an alloy structure with an average size of $7.15 \mathrm{~nm}$. To understand the properties of the bimetallic nanoparticles, it is necessary to know the distribution of the elements in the nanostructure. We have used a semi-quantitative method to analyze the HAADF-STEM images, which allowed us to measure the total intensity of the scattered electrons for each atomic column. HAADF-STEM images of the Pt-Pd bimetallic nanoparticles were compared directly with image simulations, good agreement between simulation and experimental images was found. Cyclic voltammetry studies were carried out to analyze the electrochemical behavior of the bimetallic nanoparticles.
\end{abstract}

Keywords: Nanoparticles, Pt-Pd, Scanning/transmission electron microscopy, Aberration corrected, Cyclic voltammetry

\section{Introduction}

In recent years, there has been much interest in improving the physicochemical properties of multimetallic nanoparticles. Bimetallic nanoparticles (NPs) are of interest since they lead to many interesting electrical, chemical, catalytic, and optical properties ${ }^{1}$. They are particularly important in the field of catalysis since they show superior catalytic properties than their monometallic counterparts ${ }^{2,3}$. The structure of bimetallic NPs depends mainly on the synthesis conditions and the miscibility of the two components ${ }^{4}$. The main structures of bimetallic NPs are core-shell, mixed alloys, Janus and multishell alloys configurations. Coreshell configuration consists of a shell of one type of atom surrounding a core of another, although there may be some mixing between the shells. This configuration is common to a large variety of systems $\mathrm{s}^{5-7}$. Mixed alloys configuration may be either ordered or random. In many cases random

${ }^{1}$ Article presented and selected from "The XXIV International Materials Research Congress (IMRC) 2015". Symposium 5B "Structural and Chemical Characterization of Metals, Alloys and Compounds". Cancun, Mexico August 16-20, 2015.

* e-mail: resparza@fata.unam.mx mixed configuration is often called alloyed nanoparticles. Multishell alloys configuration may present layered or onion-like alternating -A-B-A- shells ${ }^{8,9}$. However, one of the most important challenges nowadays is to know the distribution of the elements and the atomic positions in the above-mentioned configurations. Therefore, the structural characterization of the bimetallic NPs is fundamental for understanding their catalytic properties.

Pt is the most used catalytic metal in the fuel cells, mainly in direct ethanol fuel cells ${ }^{10}$; however one of the problems of the $\mathrm{Pt}$ catalyst is the poisoning by carbon monoxide ${ }^{11}$. A solution to enhancement the electrocatalytic properties is to mix the Pt with other elements or deposited on different supports ${ }^{12}$. Among Pt-based catalysts, Pt-Pd is one of the most attractive bimetallic systems due to its application as electrocatalyst in fuel cells ${ }^{13-15}$ and various applications including hydrogenation ${ }^{16-17}$ and oxidation of organic compounds ${ }^{18}$.

Several studies have been carried out about the synthesis and electrocatalytic properties of the Pt-Pd bimetallic NPs ${ }^{19-22}$; these bimetallic NPs can be produced generally 
by either successive co-reduction of $\mathrm{Pt}$ and $\mathrm{Pd}$ species or a seed-mediated growth method ${ }^{14}$, however, it important to know the chemical distribution of the elements which affects the physical and chemical properties of the nanoparticles ${ }^{23}$. Advanced electron microscopy techniques, especially scanning/ transmission electron microscopy (STEM), are indispensable for the characterization of the bimetallic NPs. The most important feature of a STEM instrument is its versatility: atomic resolution images, nano-beam diffraction patterns ${ }^{24}$, and spectroscopy data can be obtained either simultaneously or sequentially from the same region of the specimen ${ }^{25}$. Also, STEM has the potential to achieve atomic resolution images in three dimensions using electron tomography ${ }^{26}$. Since the development of spherical aberration (Cs) correctors $^{27}$, the resolution of transmission electron microscopy (TEM) and STEM has been greatly improved reaching sub-Angstrom levels. The correction of $\mathrm{Cs}$ is achieved by incorporating a separate aberration corrector, containing non-round elements, into the electron optical system to eliminate the Cs of the round objective lens. The Cs-correction allows the lens to be used with wider convergence angles of electron waves; thus, the probe size can be decreased according to the principle of Fourier transform, and probe current becomes larger than that in conventional STEM ${ }^{28}$. Using Cs-correction in a STEM instrument, it is possible to obtain atomic resolution of high-angle annular dark field (HAADF)-STEM images with high spatial resolution that can be used in the qualitative and quantitative Z-contrast analysis. When the atomic numbers of the sample have large differences, Z-contrast can be interpreted directly from the image.

It is important to carry out images simulations based on the dynamical theory of electron diffraction for the quantitative interpretation of experimental HAADF-STEM images. Multislice method ${ }^{29}$ is one of the principal methods used for dynamical simulations of HAADF-STEM images. When HAADF-STEM image simulations are carried out, all the experimental conditions of the electron microscope are taken into account, such as: acceleration voltage, defocus, coma, astigmatism, spherical aberration, chromatic aberration, probe size, convergence angle, annular detector range, and specimen thickness. In addition to the quantitative analysis of HAADF-STEM image simulations the elastic scattering and the incoherent thermal diffuse scattering (TDS) are necessary to be considered ${ }^{30}$ and also the map of the annular dark field detector recorded as a function of the position of the scanned incident electron probe ${ }^{31}$. The contribution of Bragg reflections is minimized and TDS becomes the most important contribution to image intensity ${ }^{32}$. For semiqualitative interpretation of experimental HAADF-STEM images, intensities ratios between different atom columns or intensity line profiles can be used. However, for quantitative interpretation of the HAADF-STEM images, determination of the chemical composition of individual atom columns based on measured intensity and the atomic thickness are a challenge. The atomic and background intensities should be measured for comparing the experimental and simulated images.

In this work, a chemical reduction process was used to synthesize Pt-Pd bimetallic NPs with alloy structure. The structural characterization was carried out by parallel beam $\mathrm{X}$-Ray diffraction, scanning and scanning/transmission electron microcopy. A semi-quantitative method to analyze HAADF-STEM images was used, which allowed us to measure the total intensity of the scattered electrons for each atomic column and to know the distribution of the elements in the nanoparticle. Finally, electrochemical properties of the as-prepared nanoparticles were measured using cyclic voltammetry.

\section{Experimental Procedure}

\subsection{Synthesis}

The Pt-Pd alloy bimetallic nanoparticles were produced by the reduction of Chloroplatinic acid hexahydrate $\left(\mathrm{H}_{2} \mathrm{PtCl}_{6} \cdot 6 \mathrm{H}_{2} \mathrm{O}, 50 \mathrm{mM}\right)$ with sodium borohydride $\left(\mathrm{NaBH}_{4}\right)$ and then by adding of Potassium tetrachloropalladate $\left(\mathrm{K}_{2} \mathrm{PdCl}_{4}\right.$, $50 \mathrm{mM}$ ). All solutions were dissolved in deionized water. In order to stabilize the bimetallic nanoparticles, a solution of polyvinylpyrrolidone (PVP) at constant concentration of $50 \mathrm{mM}$ was prepared and mixed during the reaction. The reducing agent $\left(\mathrm{NaBH}_{4}\right)$ was added drop wise at a concentration of $0.2 \mathrm{M}$.

\subsection{Characterization}

Pt-Pd alloy bimetallic nanoparticles were characterized as colloidal solution and as nanoparticles supported on Vulcan carbon. Copper grids with carbon film were prepared with a drop of the colloidal solution for STEM images.

The samples were characterized by scanning electron microscopy (SEM) using the Hitachi SU8230 cold field emission (CFE) SEM/STEM microscope, which is equipped with detectors that allow the simultaneous recording of various electron signals and energies.

Also, the samples were analyzed using aberrationcorrected STEM with a Jeol ARM200F (200 kV) FEG-TEM/ STEM microscope equipped with a CEOS Cs-corrector on the illumination system. High-angle annular dark field scanning transmission electron microscopy (HAADF-STEM) images were obtained by aberration-corrected STEM. The probe current used in STEM mode was $23.2 \mathrm{pA}$ using a condenser lens aperture size of 40 microns. The HAADF images were registered using a camera length of $80 \mathrm{~mm}$ and a collection angle of $50-180 \mathrm{mrad}$. In order to reduce the noise of the images recorded, the images were filtered using the Richardson-Lucy/Maximum Entropy algorithm ${ }^{33}$. 
Energy-dispersive X-Ray spectroscopy (EDS) was performed by means of Oxford XMax 80 EDS Spectrometer.

Parallel beam X-Ray diffraction (XRD) analysis of the bimetallic nanoparticles was carried out on a Rigaku Ultima $\mathrm{IV}^{\circledR} \mathrm{X}$-Ray diffractometer equipped with a $\mathrm{Cu} \mathrm{K} \alpha$ source $(\lambda=0.154 \mathrm{~nm})$ at $40 \mathrm{kV}$ and $30 \mathrm{~mA}$. The $2 \theta$ angular region between 10 and $80^{\circ}$ is recorded at a scan rate of $1 \%$ step.

\subsection{Image simulations}

HAADF-STEM image simulations have been performed using the QSTEM software package ${ }^{34}$, which uses the multislice algorithm based on the physical optics theory of Cowley and Moodie ${ }^{29}$. The QSTEM code is based on the methods described by E.J. Kirkland ${ }^{35}$. Table 1 shows the parameters considered for the simulation correspond to the experimental conditions of the microscope.

Table 1. Experimental parameters used to HAADF-STEM image simulations.

\begin{tabular}{lc}
\hline Acceleration voltage & $200 \mathrm{kV}$ \\
\hline Defocus $C_{1}$ & $-1.9 \mathrm{~nm}$ \\
Coma $B_{2}$ & $75.85 \mathrm{~nm}$ \\
Threefold astigmatism $A_{2}$ & $33.75 \mathrm{~nm}$ \\
Spherical aberration $C_{3}$ & $1 \mu \mathrm{m}$ \\
Star aberration $S_{3}$ & $0.515 \mu \mathrm{m}$ \\
Fourfold astigmatism $A_{3}$ & $0.089 \mu \mathrm{m}$ \\
Coma 5th order $B_{4}$ & $25.44 \mu \mathrm{m}$ \\
Fivefold astigmatism $A_{4}$ & $28.92 \mu \mathrm{m}$ \\
Spherical aberration 5 th order $C_{5}$ & $0.74 \mathrm{~mm}$ \\
Probe size & $1 \AA$ \\
Camera length & $80 \mathrm{~mm}$ \\
HAADF detector range & $50-180 \mathrm{mrad}$ \\
\hline
\end{tabular}

\subsection{Electrochemical measurement}

Cyclic voltammograms were collected in acidic media $\left(0.5 \mathrm{M} \mathrm{H}_{2} \mathrm{SO}_{4}\right)$ to evaluate the electrochemical response of $\mathrm{PtPd} / \mathrm{C}$ and $\mathrm{Pt} / \mathrm{C}$ electrocatalysts. Electrochemical experiments were performed in a three-electrode electrochemical cell with a BioLogic VSP Potentiostat. Glassy carbon electrodes $\left(\mathrm{Basi}^{\circledR}\right.$ ) with $\mathrm{Hg} / \mathrm{Hg}_{2} \mathrm{SO}_{4}$ saturated in $\mathrm{K}_{2} \mathrm{SO}_{4}$ for the acidic media and a graphite rod were used as the working, reference and counter electrodes, respectively. Ink containing the electrocatalyst was deposited on a glassy carbon electrode (Basi MF-2012, $3.0 \mathrm{~mm} \mathrm{Ø).} \mathrm{The} \mathrm{ink} \mathrm{was} \mathrm{prepared} \mathrm{by}$ mixing $1 \mathrm{mg}$ of electrocatalyst and $15 \mu \mathrm{L}$ of Nafion ( $5 \% \mathrm{wt}$. ElectroChem ${ }^{\circledR}$ ) into $75 \mu \mathrm{L}$ of isopropanol. The voltammograms of the profiles in acid media for the electrocatalysts were obtained at $50 \mathrm{mV} \mathrm{s}^{-1}$ scan ratio.

\section{Results and Discussion}

The properties of the bimetallic nanoparticles (NPs) are determined not only by their size, morphology and structure, also by the distribution of their elements. Hence an adequate structural and chemical characterization of the bimetallic NPs is essential. Parallel beam X-Ray diffraction (XRD) measurements of the samples were carried out to determine the structure of the nanoparticles, which could consist of a mixture of $\mathrm{Pt}$ and $\mathrm{Pd}$ individual elements, or Pt-Pd nanoparticles with alloy structure. Figure 1a shows the XRD pattern of the Pt-Pd alloy bimetallic nanoparticles, the XRD peaks at $40.05^{\circ}, 46.59^{\circ}$, and $67.90^{\circ}$ correspond to (111), (200) and (220) plane, respectively, which could match with the face-centered-cubic (fcc) structure and indicates the PtPd alloy formation (JCPDF 65-6418). The crystal size was evaluated from the XRD data using the Debye-Scherrer equation and the (111) plane diffraction, obtaining an average crystal size of $5 \mathrm{~nm}$. It is important to mention that the (111) plane of the PtPd alloy is close to (111) plane of the Pd (40.119 ${ }^{\circ}$, JCPDF 46-1043) (Figure 1b); therefore as the lattice parameter is very similar, it is necessary to analyze the samples with other techniques as electron microscopy.
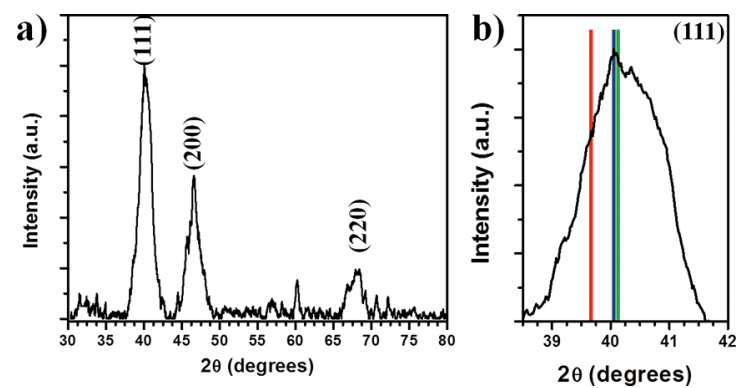

Figure 1. a) XRD pattern of the Pt-Pd alloy bimetallic nanoparticles and $b$ ) diffraction peak of (111) plane in comparison with XRD reference data of Pt (red), PtPd alloy (blue) and Pd (green).

Nowadays, one of the most important techniques used to characterize the bimetallic NPs is the scanning/transmission electron microcopy (STEM). Images with Z-contrast and highresolution can be acquire using high-angle annular dark field (HAADF)-STEM with spherical aberration (Cs)-corrected ${ }^{36}$. Figure 2a shows some low magnification HAADF-STEM images of the Pt-Pd alloy bimetallic NPs. These bimetallic NPs have a particle size about $7.15 \mathrm{~nm}$ (Figure 2b), which is close to the crystal size obtained by XRD. It is interesting to observe that the majority of the nanoparticles produced by this synthesis process did not present agglomeration and also it is clear from this image that the contrast of the image shows that the nanoparticles have an alloy structure, where the atoms of $\mathrm{Pd}$ and $\mathrm{Pt}$ have a random distribution, and the main crystallographic structure of the bimetallic NPs is fcc- 
like, however, with these images is not possible determine adequately the distribution of the elements, given that the image resolution is not sufficient.
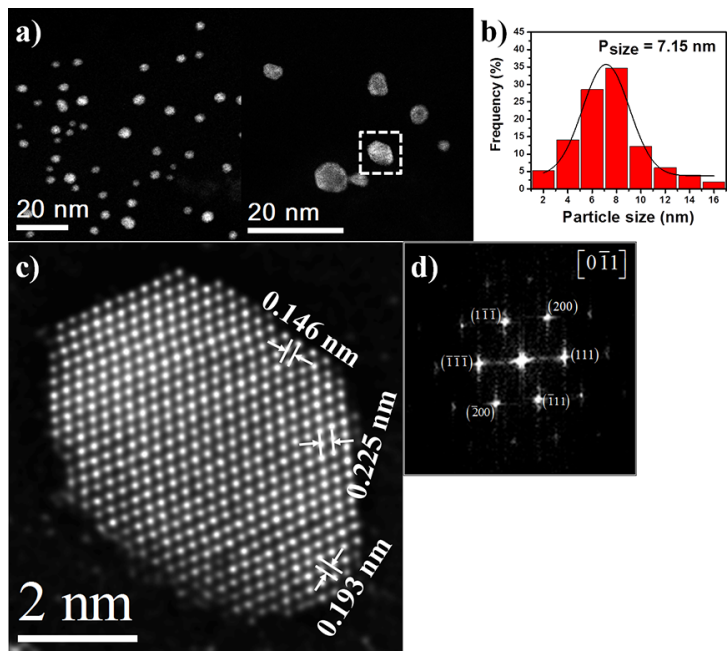

Figure 2. a) Low-magnification HAADF-STEM images, b) particle size distribution plot, c) High-resolution HAADF-STEM image of PtPd alloy bimetallic nanoparticle, and d) FFT shows the [0-11] zone axis.

To determine the distribution of the elements with high precision, it is necessary to acquire images with higher resolution. Figure $2 c$ shows a high-resolution HAADF-STEM image of a Pt-Pd alloy bimetallic nanoparticle labeled with square in Figure 2a. From the figure, d-spacings 0.225, 0.193 and $0.146 \mathrm{~nm}$ were obtained. Such d-spacings correspond to (111), (200) and (022) crystalline planes respectively of the Pd-Pt face-centered-cubic crystal structure with $\mathrm{a}_{0}=0.3896 \mathrm{~nm}$ (JCPDF 65-6418). Fast Fourier transform (FFT) shows the main reflections confirming the [0-11] zone axis (Figure 2d). The majority of the bimetallic NPs analyzed showed the same face-centered-cubic crystal structure and the nanoparticles were associated mainly with cuboctahedral morphology with dominantly exposed $\{100\}$ and $\{111\}$ facets. It is interesting to observe that in some facets monoatomic-layer islands can be found. The high resolution HAADF-STEM image shows a slight distortion in the crystalline planes, which are related with the effect of the scanning beam over the specimen. These distortions are showed as parallel fringes in the scanning direction and sometimes are misinterpreted as lattice distortions; however are the distortions arising from environmental or instrumental instabilities ${ }^{37}$. It is interesting to observe in this figure the difference in contrast obtained from the Pt and Pd atoms. The contrast in the HAADF-STEM images is associated with the atomic number $\mathrm{Z}$ of the elements in the sample, therefore, the strong brightness corresponds to the heaviest element $(\mathrm{Pt})$ and the low brightness corresponds to the lightest element
(Pd). From the image clearly is observable the distribution of the Pt and Pd elements in the bimetallic nanoparticle.

Figure 3 shows the energy-dispersive spectroscopy (EDS) analysis of the elemental composition of the Pt-Pd alloy bimetallic NPs. The EDS spectrum (Figure $3 b$ ) shows the presence of $\mathrm{Pt}$ and $\mathrm{Pd}(\mathrm{Cu}$ and $\mathrm{O}$ are associated with the grid and the dispersion medium respectively). The elemental composition of the $\mathrm{Pt}$ and $\mathrm{Pd}$ shows an atomic ratio close to $(1: 1)$. The figure displays the elemental mapping images for the bimetallic nanoparticle. The red and green color images are associated with the $\mathrm{Pt}$ and Pd elements, respectively. The mapping images were acquired using the L-line spectra of the elements. Also the overlap image is shown. It can be observed that the elements have an inhomogeneous distribution in the nanoparticle, where exist zones with high content of Pt and similar zones with high content of Pd. This indicates that the elements are localized in the nanoparticle in random form. Therefore, this confirms the alloy structure of the bimetallic nanoparticle.
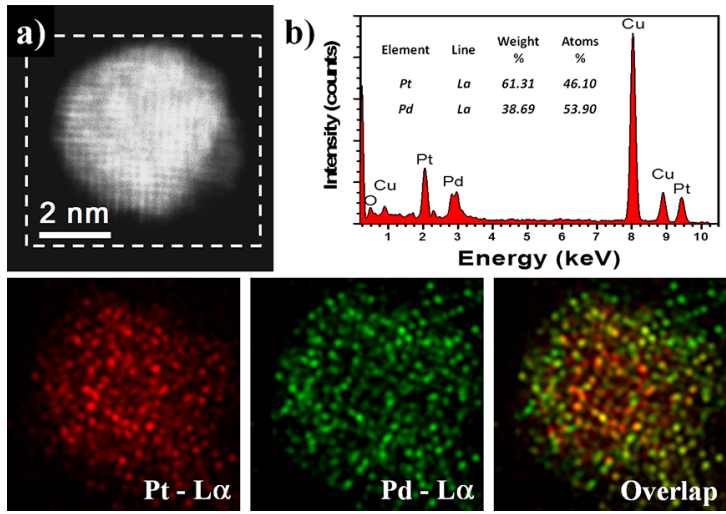

Figure 3. a) HAADF-STEM image of a Pt-Pd alloy bimetallic nanoparticle, b) EDS of the plotted area and EDS mapping of Pt, $\mathrm{Pd}$ and overlap elements.

The main advantage of HAADF-STEM images over high-resolution transmission electron microscopy (HR-TEM) images is its incoherence; there are no contrast changes as can been seen in HR-TEM due to the phase contrast of the imaging ${ }^{38}$. The contrast obtained in the HAADF-STEM images is dominated by Rutherford scattering; in this way the contribution of the Bragg reflections is minimized and incoherent thermal diffuse scattering (TDS) becomes the prevailing contribution to the image intensity ${ }^{39}$. The scattered intensity scale is associated mainly with the atomic number $\mathrm{Z}$ of the elements in the material. In this case, the difference between the atomic number of the elements $\mathrm{Pt}(Z=78)$ and $\mathrm{Pd}(Z=46)$ makes possible to observe notable differences in the contrast of the image. Some experiments and calculations reveal that the $Z$ dependence of the signal exponent is around 1.6-1.8 $8^{40}$. To visualize a better contrast, Apply_CLUT (color look-up table) script from D. R. G. Mitchell was used in the high resolution HAADF-STEM image (Figure 4a). This 
script is useful for highlighting subtle intensity variations, which are much more evident in color temperature scale than grayscale. The image clearly shows differences in contrast intensity and some atomic columns are brighter than others. However, the brightness intensity of HAADF-STEM images of nanoparticles are almost insensitive to defocus and/or thickness changes due to the few number of atomic columns ${ }^{41}$; therefore the brightness intensity of the image is directly related with the atomic number of the elements. It is clear from the brightness intensity of the image that some atomic columns have a larger number of Pt atoms, since they are brighter and are indicated with a yellow color; instead, some atomic columns have a red less bright color which are associated with the Pd atoms.
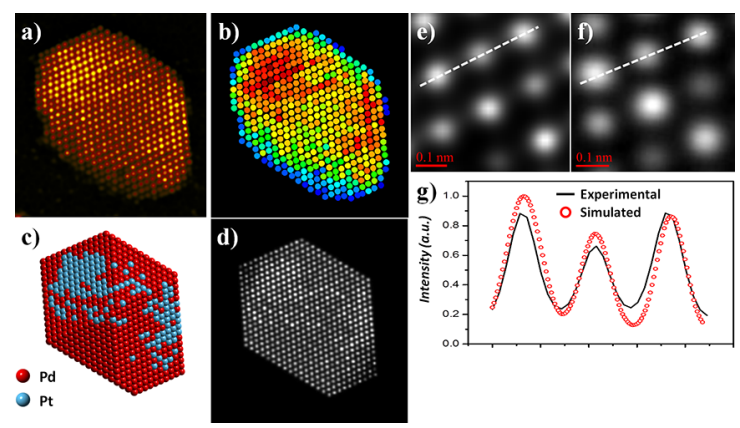

Figure 4. a) HAADF-STEM image of a Pt-Pd bimetallic nanoparticle, b) Map representing each atom by its integrated intensity, c) atomic model of the PtPd alloy nanoparticle with a cuboctahedron shape, d) simulated HAADF-STEM image of the bimetallic nanoparticle, e) and f) selected small region of the experimental and simulated images, and g) line profile across the marked in the images.

Figure $4 \mathrm{~b}$ shows the contrast intensity analysis performed using Absolute Integrator v1.2 program. The positions of all atomic columns in the experimental image were determined by normalized cross correlation after applying a RichardsonLucy filter to the image in order to reduce the noise ${ }^{33}$, which improved the accuracy of column finding. The intensity of the signal from all atomic columns in the experimental image was extracted and plotted as a color map representing integrated intensity of the atoms. It is very important to analyze in this figure that the $\mathrm{Pt}$ and $\mathrm{Pd}$ atoms are distributed randomly; it is also possible to observe some surface segregation of the Pt atoms in the bimetallic nanoparticle. The main factors influencing the surface segregation process are determined by atomic size, heat of solution of the alloy and the difference in the surface energies of the pure metal ${ }^{42}$. However, in the case of Pt-Pd alloys, atomic size and heat of solution effects for surface segregation can be neglected because the heat of solution of the alloy is very small; besides the Pt and Pd atoms have similar sizes. It has been reported by Monte Carlo calculations ${ }^{43}$ that the (111) facets have higher Pt concentration atoms than the (100) facets in Pt-Pd alloys with cuboctahedral shape. However, the Pt concentration in the (100) facets does not vary with respect the particle size, instead, the Pt concentration in the (111) facets decreases drastically with the particle size. In this figure the Pt concentration is mainly located in the (100) facets that could be dominated by the particle size.

To confirm the above obtained results, simulations of HAADF-STEM images have been performed. Figures $4 \mathrm{c}$ and $4 \mathrm{~d}$ show the atomic model and simulated HAADF-STEM image of a Pd-Pt bimetallic nanoparticle. The parameters used in the simulation procedure are shown in Table 1, which correspond to the experimental parameters of the electron microscope. The atomic model used for the simulated images has a particle size about $4.5 \mathrm{~nm}$ with a thickness of $6.5 \mathrm{~nm}$. From the simulated image, the brightness obtained from the different atoms is clear; display more brightness from atomic columns with major content of Pt atoms and less brightness from atomic columns with minor content of Pd. The direct matching of experimental and simulated HAADF-STEM images is carried out by the comparison of intensity profiles determined across some atomic columns. Figures $4 \mathrm{e}$ and $4 \mathrm{f}$ show a small region of the experimental and simulated HAADF-STEM images, respectively. As can be observed there is good agreement between the two images. Figure $4 \mathrm{~g}$ shows the intensity line profiles across the atomic column from the experimental and simulated images. The line profiles of the experimental and simulated images match perfectly. A qualitative interpretation of atomic-resolution HAADFSTEM images is usually straightforward since the atomic columns with the higher average atomic number, always possess a higher intensity compared to the atomic columns with the lower average atomic number. However, to obtain a semi-quantitative interpretation and a good characterization of the nanoparticles, it is required image simulations and a good matching among the experimental images with the calculated ones.

Pt-Pd alloy bimetallic nanoparticles were supported on Vulcan carbon black to analyze their electrocatalytic behavior. Figure 5a shows a scanning electron microscopy (SEM) image at low voltage using backscattered electrons detected by the Top detector, which has pure Z-number contrast with less topographical information. After that the nanoparticles were supported on the carbon black, it is evident that the nanoparticles were uniformly dispersed, and agglomeration was not observed, which it is very important because of the particle size and morphology are similar to those before load on the carbon. Figure 5b shows a SEM image generated using low energy secondary electrons detected by using the same Top detector but now in deceleration mode. As can be observed, the bimetallic nanoparticles with particle size between 5 to $10 \mathrm{~nm}$ are localized on the Vulcan carbon surface; also it is important to observe the porosity of the Vulcan carbon, which help to enhanced open circuit voltage of fuel cells, diminish the crossover, and expand the active area of the catalysts, leading to improvements in transport of fuel and by-products ${ }^{44}$. 

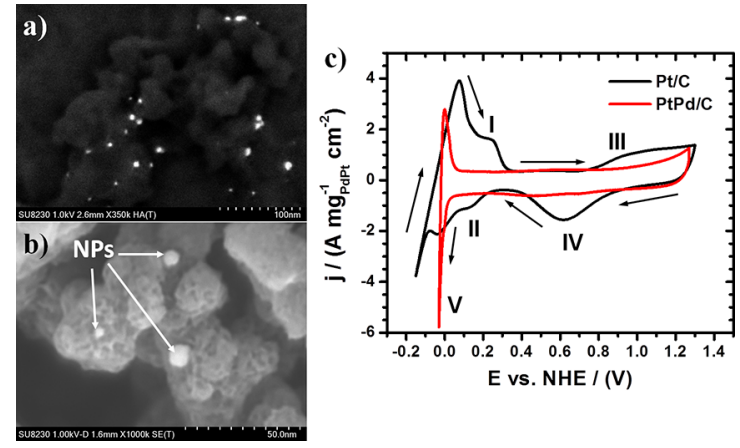

Figure 5. a) and b) SEM images of the PtPd alloy nanoparticles supported on Vulcan carbon black obtained with the backscattered and secondary electrons detector at low voltage, and c) cyclic voltammograms of the $\mathrm{PtPd} / \mathrm{C}$ and commercial $\mathrm{Pt} / \mathrm{C}$ electrocatalysts.

Figure $5 \mathrm{c}$ shows the electrochemical response of the $\mathrm{PtPd} / \mathrm{C}$ electrocatalyst in compare with a commercial Pt/C electrocatalyst (ETEK, $20 \% \mathrm{wt}$ ) in acidic media $\left(0.5 \mathrm{M} \mathrm{H}_{2} \mathrm{SO}_{4}\right)$. For the commercial $\mathrm{Pt} / \mathrm{C}$ it can observe different zones in their electrochemical response. The process (I) is related to the protons desorption on the electrocatalyst surface. Also, the process (II) corresponds for the protons adsorption.

$$
\begin{aligned}
& \text { I: } \mathrm{H}_{a d s} \rightarrow \mathrm{H}^{+}+\mathrm{e}^{-} \\
& \text {II: } \mathrm{H}^{+}+\mathrm{e}^{-} \rightarrow \mathrm{H}_{a d s}
\end{aligned}
$$

The processes (III) and (IV) describe the formation and reduction of platinum oxides, respectively.

$$
\begin{aligned}
& \text { III: } \mathrm{Pt}^{0}+\mathrm{H}_{2} \mathrm{O} \rightarrow \mathrm{Pt}-\mathrm{H}_{2} \mathrm{O}_{a d s} \rightarrow \mathrm{Pt}-\mathrm{OH}_{a d s}+\mathrm{H}^{+}+\mathrm{e}^{-} \\
& \text {IV: } \mathrm{Pt}-\mathrm{OH}_{a d s}+\mathrm{H}^{+}+\mathrm{e}^{-} \rightarrow \mathrm{Pt}-\mathrm{H}_{2} \mathrm{O}_{a d s} \rightarrow \mathrm{Pt}^{0}+\mathrm{H}_{2} \mathrm{O}
\end{aligned}
$$

For the PtPd/C electrocatalyst, the formation (III) and reduction (IV) of platinum oxides are not present and are directly related with the adsorption/desorption, because the processes (I) and (II) does not appear in their electrochemical response. A similar behavior occurs with the existing palladium in the $\mathrm{PtPd} / \mathrm{C}$ electrocatalyst.

The hydrogen evolution (process V) starts at -0.023 $\mathrm{V}$ vs. NHE and finishes at $0 \mathrm{~V}$ vs. NHE for the PtPd/C electrocatalyst and are more positive values in compare with the commercial Pt/C (-0.111 V vs. NHE). The result suggests that the $\mathrm{PtPd} / \mathrm{C}$ electrocatalyst is a viable option to use as anodic material for proton-exchange membrane fuel cells (PEMFC) using hydrogen as fuel.

\section{Conclusions}

A semi-quantitative analysis of Cs-corrected HAADF-STEM images of Pd-Pt bimetallic nanoparticles was systematically investigated. The method for the calculation of HAADF-STEM images used in this work is dependent of atomic number of the elements. It is shown that HAADF-STEM technique is a sensitive method for the identification of the atoms in bimetallic nanoparticles. Simulations of HAADF-STEM images can suggest the atomic positions and the intensities of the $\mathrm{Pt}$ and Pd elements. The interpretation of atomic column intensities was performed using a comparison between experimental and their corresponding simulated HAADF-STEM images. The average size of the Pt-Pd bimetallic nanoparticles synthesized by chemical reduction method was around $7.15 \mathrm{~nm}$. This process is an excellent technique to synthesize bimetallic nanoparticles with smaller sizes and narrow size distribution, which to improve their physical and chemical properties. For many applications Pt has better catalytic activity than Pd, however, Pt-Pd bimetallic nanoparticles where Pt atoms are located in some facets are attractive in many catalytic applications. Furthermore, PtPd/C electrocatalyst showed a better potential for the beginning of the hydrogen evolution compared to commercial $\mathrm{Pt} / \mathrm{C}$.

\section{Acknowledgements}

The authors would like to acknowledge to the PAPIITDGAPA for financial support with grant IN110113. Thanks to A. García-Ruiz for their help in the synthesis of nanomaterials. Also, the authors acknowledge the Laboratorio Avanzado de Nanoscopía Electrónica "LANE" at the CINVESTAVZacatenco.

\section{References}

1. Cho SJ, Ryoo R. Design of bimetallic nanoparticles. International Journal of Nanotechnology. 2006;3(2-3):194-215.

2. Garcia-Gutierrez DI, Gutierrez-Wing CE, Giovanetti L, RamalloLópez JM, Requejo FG, Jose-Yacaman M. Temperature Effect on the Synthesis of Au-Pt Bimetallic Nanoparticles. The Journal of Physical Chemistry B. 2005;109(9):3813-3821.

3. Devarajan S, Bera P, Sampath S. Bimetallic nanoparticles: A single step synthesis, stabilization, and characterization of $\mathrm{Au}-\mathrm{Ag}, \mathrm{Au}-\mathrm{Pd}$, and $\mathrm{Au}-\mathrm{Pt}$ in sol-gel derived silicates. Journal of Colloid and Interface Science. 2005;290(1):117-129.

4. Santhanalakshmi J, Venkatesan P. Mono and bimetallic nanoparticles of gold, silver and palladium-catalyzed NADH oxidation-coupled reduction of Eosin-Y. Journal of Nanoparticle Research. 2011;13(2):479-490.

5. Chaudhuri RG, Paria S. Core/Shell Nanoparticles: Classes, Properties, Synthesis Mechanisms, Characterization, and Applications. Chemical Reviews. 2012;112(4):2373-2433.

6. Mayoral A, Llamosa D, Huttel Y. A novel Co@Au structure formed in bimetallic core@shell nanoparticles. Chemical Communications. 2015;51(40):8442-8445.

7. Mayoral A, Mejía-Rosales S, Mariscal MM, Pérez-Tijerina E, José-Yacamán M. The Co-Au interface in bimetallic nanoparticles: a high resolution STEM study. Nanoscale. 2010;2(12):2647-2651. 
8. Ferrer D, Torres-Castro A, Gao X, Sepúlveda-Guzmán S, Ortiz-Mendez U, José-Yacamán M. Three-Layer Core/Shell Structure in Au-Pd Bimetallic Nanoparticles. Nano Letters. 2007;7(6):1701-1705.

9. Deepak FL, Casillas-Garcia G, Esparza R, Barron H, Jose-Yacaman $\mathrm{M}$. New insights into the structure of PdAu nanoparticles as revealed by aberration-corrected STEM. Journal of Crystal Growth. 2011;325(1):60-67.

10. Wang Y, Zou S, Cai WB. Recent Advances on Electro-Oxidation of Ethanol on Pt- and Pd-Based Catalysts: From Reaction Mechanisms to Catalytic Materials. Catalysts. 2015;5(3):1507-1534.

11. Li Q, He R, Gao JA, Jensen JO, Bjerrum NJ. The CO Poisoning Effect in PEMFCs Operational at Temperatures up to $200^{\circ} \mathrm{C}$. Journal of The Electrochemical Society. 2003;150(12):A1599-A1605.

12. Zhang K, Yang W, Ma C, Wang Y, Sun C, Chen Y, et al. A highly active, stable and synergistic $\mathrm{Pt}$ nanoparticles $/ \mathrm{Mo}_{2} \mathrm{C}$ nanotube catalyst for methanol electro-oxidation. NPG Asia Materials. 2015;7:e153.

13. Huang R, Wen YH, Zhu ZZ, Sun SG. Pt-Pd Bimetallic Catalysts: Structural and Thermal Stabilities of Core-Shell and Alloyed Nanoparticles. The Journal of Physical Chemistry C. 2012;116(15):8664-8671.

14. Wang L, Nemoto Y, Yamauchi Y. Direct Synthesis of SpatiallyControlled Pt-on-Pd Bimetallic Nanodendrites with Superior Electrocatalytic Activity. Journal of The American Chemical Society. 2011;133(25):9674-9677.

15. Lv JJ, Zheng JN, Wang YY, Wang AJ, Chen LL, Feng JJ. A simple one-pot strategy to platinum-palladium@palladium core-shell nanostructures with high electrocatalytic activity. Journal of Power Sources. 2014;265:231-238.

16. Li X, Li B, Cheng M, Du Y, Wang X, Yang P. Catalytic hydrogenation of phenyl aldehydes using bimetallic $\mathrm{Pt} / \mathrm{Pd}$ and $\mathrm{Pt} /$ Au nanoparticles stabilized by cubic silsesquioxanes. Journal of Molecular Catalysis A: Chemical. 2008;284(1-2):1-7.

17. Datta KJ, Datta KKR, Gawande MB, Ranc V, Čépe K, Malgras V, et al. Pd@Pt Core-Shell Nanoparticles with Branched Dandelion-like Morphology as Highly Efficient Catalysts for Olefin Reduction. Chemistry A European Journal. 2016;22(5):15771581 .

18. Morlang A, Neuhausen U, Klementiev KV, Schütze FW, Miehe $\mathrm{G}$, Fuess H, et al. Bimetallic Pt/Pd diesel oxidation catalysts: Structural characterisation and catalytic behavior. Applied Catalysis B: Environmental. 2005;60(3-4):191-199.

19. Long NV, Thi CM, Nogami M, Ohtaki M. Pt and Pd Based Catalysts with Novel Alloy and Core-Shell Nanostructures for Practical Applications in Next Fuel Cells: Patents and Highlights. Recent Patents on Materials Science. 2012;5(3):175-190.

20. Lai J, Luque R, Xu G. Recent Advances in the Synthesis and Electrocatalytic Applications of Platinum-Based Bimetallic Alloy Nanostructures. Chem CatChem. 2015;7(20):3206-3228.

21. Sun L, Wang H, Eid K, Alshehri SM, Malgras V, Yamauchi Y, et al. One-Step Synthesis of Dendritic Bimetallic PtPd Nanoparticles on Reduced Graphene Oxide and Its Electrocatalytic Properties. Electrochimica Acta. 2016;188:845-851.
22. Eid K, Malgras V, He P, Wang K, Aldalbahi A, Alshehri SM, et al. One-step synthesis of trimetallic Pt-Pd-Ru nanodendrites as highly active electrocatalysts. RSC Advances. 2015;5(39):31147-31152.

23. Bhattarai N, Khanal S, Velazquez-Salazar JJ, Jose-Yacaman M. Advanced Electron Microscopy in the Study of Multimetallic Nanoparticles. In: Deepak FL, Mayoral A, Arenal R, eds. Advanced Transmission Electron Microscopy: Applications to Nanomaterials. Basel: Springer Internal Publishing; 2015. p. 59-91.

24. Bahena D, Bhattarai N, Santiago U, Tlahuice A, Ponce A, Bach $\mathrm{SBH}$, et al. STEM Electron Diffraction and High-Resolution Images Used in the Determination of the Crystal Structure of the $\mathrm{Au}_{144}(\mathrm{SR})_{60}$ Cluster. The Journal of Physical Chemistry Letters. 2013;4(6):975-981.

25. Liu J. Scanning transmission electron microscopy and its application to the study of nanoparticles and nanoparticle systems. Journal of Electron Microscopy (Tokio). 2005;54(3):251-278.

26. Van Aert S, Batenburg KJ, Rossell MD, Erni R, Van Tendeloo G Three-dimensional atomic imaging of crystalline nanoparticles. Nature. 2011;470(7334):374-377.

27. Haider M, Uhlemann S, Schwan E, Rose H, Kabius B, Urban K. Electron microscopy image enhanced. Nature. 1998;392(6678):768-769.

28. Tanaka N. Present status and future prospects of spherical aberration corrected TEM/STEM for study of nanomaterials. Science and Technology of Advanced Materials. 2008;9(1):014111.

29. Cowley JM, Moodie AF. The scattering of electrons by atoms and crystals. I. A new theoretical approach. Acta Crystallographica. 1957;10:609-619.

30. Pizarro J, Galindo PL, Guerrero E, Yáñez A, Guerrero MP, Rosenauer A, et al. Simulation of high angle annular dark field scanning transmission electron microscopy images of large nanostructures. Applied Physics Letters. 2008;93(15):153107.

31. LeBeau JM, Stemmer S. Experimental quantification of annular dark-field images in scanning transmission electron microscopy. Ultramicroscopy. 2008;108(12):1653-1658.

32. Kotaka Y. Essential experimental parameters for quantitative structure analysis using spherical aberration-corrected HAADFSTEM. Ultramicroscopy. 2010;110(5):555-562.

33. Ishizuka K. Deconvolution Processing in Analytical STEM: Monochromator for EELS and Cs-Corrector for STEM-HAADF. Microscopy and Microanalysis. 2005;11(Suppl 2):1430-1431.

34. Koch CT. Determination of Core Structure Periodicity and Point Defect Density along Dislocations. [Thesis]. Tempe: Arizona State University; 2002.

35. Kirkland EJ. Advanced Computing in Electron Microscopy. $2^{\text {nd }}$ ed. New York: Springer; 2010.

36. Nellist PD, Pennycook SJ. The principles and interpretation of annular dark-field Z-contrast imaging. Advances in Imaging and Electron Physics. 2000;113:147-203.

37. Jones L, Nellist PD. STEM Image Post-processing for Instability and Aberration Correction for Transfer Function Extension. Journal of Physics: Conference Series. 2012;371:012001. 
38. E H, Nellist PD, Lozano-Perez S, Ozkaya D. Towards quantitative analysis of core-shell catalyst nanoparticles by aberration corrected high angle annular dark field STEM and EDX. Journal of Physics: Conference Series. 2010;241:012067.

39. Muller DA, Edwards B, Kirkland EJ, Silcox J. Simulation of thermal diffuse scattering including a detailed phonon dispersion curve. Ultramicroscopy. 2001;86(3-4):371-380.

40. Erni R, Heinrich H, Kostorz G. Quantitative characterization of chemical inhomogeneities in Al-Ag using high-resolution Z-contrast STEM. Ultramicroscopy. 2003;94(2):125-133.

41. MacArthur KE, Jones LB, Lozano-Perez S, Ozkaya D, Nellist PD. Structural quantification of nanoparticles by HAADF STEM. Journal of Physics: Conference Series. 2014;522:012061.
42. Wang G, Van Hove MA, Ross PN, Baskes MI. Quantitative prediction of surface segregation in bimetallic Pt-M alloy nanoparticles $(\mathrm{M}=\mathrm{Ni}, \mathrm{Re}, \mathrm{Mo})$. Progress in Surface Science. 2005;79(1):28-45.

43. Duan Z, Wang G. Monte Carlo simulation of surface segregation phenomena in extended and nanoparticle surfaces of Pt-Pd alloys. Journal of Physics: Condensed Matter. 2011;23(47):475301.

44. Lee Y, Kim TK, Choi YS. Effect of Porosity in Catalyst Layers on Direct Methanol Fuel Cell Performances. Fuel Cells. 2013;13(2):173-180. 